\title{
Computation of Independent Contact Regions for Grasping 3-D Objects
}

\author{
Máximo A. Roa, Student Member, IEEE, and Raúl Suárez, Member, IEEE
}

\begin{abstract}
Precision grasp synthesis has received a lot of attention in past few last years. However, real mechanical hands can hardly assure that the fingers will precisely touch the object at the computed contact points. The concept of independent contact regions (ICRs) was introduced to provide robustness to finger positioning errors during an object grasping: A finger contact anywhere inside each of these regions assures a force-closure grasp, despite the exact contact position. This paper presents an efficient algorithm to compute ICRs with any number of frictionless or frictional contacts on the surface of any 3-D object. The proposed approach generates the independent regions by growing them around the contact points of a given starting grasp. A two-phase approach is provided to find a locally optimal force-closure grasp that serves as the starting grasp, considering as grasp quality measure the largest perturbation wrench that the grasp can resist, independently of the perturbation direction. The proposed method can also be applied to compute ICRs when several contacts are fixed beforehand. The approach has been implemented, and application examples are included to illustrate its performance.
\end{abstract}

Index Terms-Fixture, grasp, independent contact regions (ICRs), manipulation planning, multifingered hands.

\section{INTRODUCTION}

$\mathbf{M}$ ULTIFINGERED hands have become an area of great interest in robotics, mainly because they increase the flexibility and versatility of the robotic arms, allowing the robot to grasp and manipulate a large class of objects with a single end effector. The grasp planning problem associated with multifingered hands looks for the set of contact locations of the fingers on the object surface that fulfill some desired condition. When frictional contacts are considered, the most frequently used condition is force closure (FC), i.e., that the forces applied by the fingers ensure the object immobility [1]. When the position of the contacts ensures the object immobility, the grasp is form closure; this condition is mostly used in fixture design for object inspection or in manufacturing, basically when the application requires a grasp that does not rely on friction (i.e., a total kinematic restriction).

Two types of grasps are mainly considered for multifingered hands: power grasps, which use the whole surface of the hand

Manuscript received June 29, 2008; revised November 25, 2008. First published May 8, 2009; current version published July 31, 2009. This paper was recommended for publication by Associate Editor O. Brock and Editor K. Lynch upon evaluation of the reviewers' comments. This work was supported in part by the CICYT projects DPI2007-63665 and DPI2008-02448.

M. A. Roa is with the Technical University of Catalonia, 08028 Barcelona, Spain. He is also with the Large Format Printing Division, Hewlett Packard, 08174 Sant Cugat del Valles, Barcelona, Spain (e-mail: maroag@ieee.org).

R. Suárez is with the Institute of Industrial and Control Engineering, Technical University of Catalonia, 08028 Barcelona, Spain (e-mail: raul.suarez@upc.edu).

Color versions of one or more of the figures in this paper are available online at http://ieeexplore.ieee.org.

Digital Object Identifier 10.1109/TRO.2009.2020351 to restrain the object, and precision grasps, which use only the fingertips to grasp the object. Power grasp synthesis has generally been tackled by adapting a grasp from a database of generic grasp shapes [2], [3]. Most of the theoretical work on grasp synthesis is focused on computing precision grasps; several works have presented such methods for 2-D polygonal [4], [5], irregular [6] or discrete objects [7], 3-D polyhedral objects [8], [9], objects with smooth curved surfaces [10], [11], and 3-D discrete objects [12], [13].

In general, given an object and a mechanical hand, there is more than one grasp that fulfills the force or form closure property. An optimal grasp is chosen using a quality measure, based on criteria such as geometrical considerations (e.g., the distance between the centroid of the grasp polygon and the center of mass of the object [14]), limits on the forces applied by the fingers on the object (e.g., the maximal disturbance resisted by the grasp [15]), task-oriented quality measures [16], [17], or measures associated with the hand configuration [18]. A review of grasp quality measures is provided in [19], and two overviews of robotic grasping and manipulation, including grasp synthesis, are provided in [20] and [21].

Grasp synthesis algorithms provide the contact locations for the fingers on the object surface, but real mechanical hands can hardly assure that the fingers are positioned in the exact locations due to different sources of uncertainty. To provide robustness to finger positioning errors, the computation of independent contact regions (ICRs) on the object boundary was introduced, such that if each finger is positioned on an ICR, an FC grasp is always obtained independently of the exact position of each finger [22]. The determination of ICRs was initially addressed for two frictional contacts on polygonal and polyhedral objects and with four frictionless contacts on 2-D polygonal objects [22]. The concept was extended to three-finger grasps of polygonal objects [14], to four-finger grasps of polyhedral objects [8], and to grasps with a large number of fingers on 3-D objects based on initial examples [23].

To deal with complex objects, as well as with objects whose boundary is only known at a finite set of points (e.g., the aerodynamic design of an airfoil [12]), the object surface is approximated via a triangular mesh with a high number of faces or with a set of surface points and their corresponding normal direction. The computation of ICRs for 2-D discrete objects with four contact points has been already addressed [24], and a first approach to the determination of ICRs on 3-D discrete objects has been recently tackled by the authors [25].

This paper deals with the problem of determining ICRs on a 3-D object boundary using any number $n$ of contacts (satisfying the necessary conditions: $n \geq 3$ for frictional contacts and $n \geq 7$ 
for frictionless contacts [26], [27]), such that the ICRs assure an FC grasp with a controlled minimum quality. The proposed algorithm generates the ICRs by growing them from a starting FC grasp, which is properly computed. The approach focuses on the object and does not take into account kinematic constraints imposed by the mechanical hand or gripper.

The assumptions and the required background, including the FC test and the quality measure used in the paper, are provided in Section II. Section III presents the procedure to compute a starting FC grasp, and the algorithm to obtain the ICRs is described in Section IV. Section V shows the application of the implemented approach on different objects with frictional and frictionless contacts. Finally, Section VI summarizes the work and discusses future research in this area.

\section{BASIC BACKGROUND}

\section{A. Object Model}

The approach is intended to be valid for rigid objects of any shape; thus, it is assumed that the object surface is represented by any type of mesh, i.e., a set $\Omega$ of $N$ points plus some neighboring information among them (the number of neighbors has no influence on the proposed approach). The points are described by position vectors $\boldsymbol{p}_{i}$ measured with respect to a reference system located in the center of mass (CM) of the object, and each point $\boldsymbol{p}_{i}$ has an associated surface normal direction $\hat{\boldsymbol{n}}_{i}$ pointing toward the interior of the object. $N$ is assumed to be large enough to accurately represent the object, and the points are close enough to be real neighbors not only in the physical space but also in the mesh model. With this description, each ICR is obtained as a set of neighboring points, assuming that a contact between them also generates an FC grasp.

\section{B. Contact and Force Model}

The contact between each finger and the object is considered punctual, either with or without friction. For frictional contacts, Coulomb's friction model is used, which states that in order to avoid slippage the force $\boldsymbol{f}_{i}$ applied at $\boldsymbol{p}_{\boldsymbol{i}}$ must lie inside the friction cone defined by $\boldsymbol{f}_{i}^{t} \leq \mu \boldsymbol{f}_{i}^{n}$, where $\mu$ is the friction coefficient and $\boldsymbol{f}_{i}^{t}$ and $\boldsymbol{f}_{i}^{n}$ are, respectively, the tangential and normal components of $\boldsymbol{f}_{i}$. In the 3 -D physical space, this model is nonlinear, and to simplify it, the friction cone is linearized using an $m$-side polyhedral convex cone (the more sides, the better the approximation, but the higher the computational cost of dealing with the linearized cone). Thus, by representing the unitary vector along the $j$ th edge of the convex cone at the $i$ th contact with $\hat{\boldsymbol{n}}_{i j}$, the grasping force is given by

$$
\boldsymbol{f}_{i}=\sum_{j=1}^{m} \alpha_{i j} \hat{\boldsymbol{n}}_{i j}, \quad \alpha_{i j} \geq 0 .
$$

The force $\boldsymbol{f}_{i}$ applied on the object at $\boldsymbol{p}_{i}$ generates a torque $\boldsymbol{\tau}_{i}=\boldsymbol{p}_{i} \times \boldsymbol{f}_{i}$ with respect to CM. The variables $\boldsymbol{f}_{i}$ and $\boldsymbol{\tau}_{i}$ are grouped together in a wrench vector given by $\boldsymbol{\omega}_{i}=\left(\boldsymbol{f}_{i} \boldsymbol{\tau}_{i} / \rho\right)^{T}$, where $\rho$ is a parameter with units of length used to adjust the metric of the wrench space (the appendix gives further details on $\rho$ ).
In order to simplify future explanations, the following abbreviations will be used throughout the paper for wrenches applied at $\boldsymbol{p}_{\boldsymbol{i}}$ :

1) $\boldsymbol{\omega}_{i}$ : wrench generated by a unitary force $\boldsymbol{f}_{i}$ orthogonal to the object surface, i.e., $\boldsymbol{f}_{i}=\hat{\boldsymbol{n}}_{i}$;

2) $\boldsymbol{\omega}_{i j}$ : wrench generated by a unitary force $\boldsymbol{f}_{i}$ along an edge of the linearized friction cone, i.e., $\boldsymbol{f}_{i}=\hat{\boldsymbol{n}}_{i j}\left(\boldsymbol{\omega}_{i j}\right.$ is called a primitive wrench).

Since each $\boldsymbol{p}_{\boldsymbol{i}}$ is associated with the wrenches $\boldsymbol{\omega}_{i}$ and $\boldsymbol{\omega}_{i j}$, a grasp defined by the set of contact points $C=\left\{\boldsymbol{p}_{1}, \ldots, \boldsymbol{p}_{n}\right\}$ is associated with the sets $G=\left\{\boldsymbol{\omega}_{1}, \ldots, \boldsymbol{\omega}_{n}\right\}$ and $W=$ $\left\{\boldsymbol{\omega}_{11}, \ldots, \boldsymbol{\omega}_{1 m}, \ldots, \boldsymbol{\omega}_{n 1}, \ldots, \boldsymbol{\omega}_{n m}\right\}$. Thus, $G$ and $W$ will be used as representative sets of a grasp (actually, a given set $G$ could be associated with more than one set of points on the object surface, but the existence of $G$ satisfying the desired grasp requirements ensures the existence of at least one set of points $C$ that also do so).

Frictionless contacts, which are usually considered in fixturing where a total kinematic restriction is desired, only allow grasping forces to be applied in the direction normal to the object surface. For the given friction model, it can be assumed that the linearized friction cone is described by only one vector $\hat{\boldsymbol{n}}_{i 1}=\hat{\boldsymbol{n}}_{i}$. Therefore, the aforementioned abbreviations are valid for frictional as well as for frictionless contacts, and so are the algorithms presented later in this paper.

\section{FC and Grasp Quality}

A necessary and sufficient condition for the existence of an FC grasp is that the origin $O$ of the wrench space lies strictly inside the convex hull of $W$ [28], which, from now on, is represented as $\mathrm{CH}(W)$. Several FC tests based on this condition have been proposed, for instance, solving linear optimization problems [10], [29] or using collision checks [30]. Another approach is based on linear matrix inequalities that efficiently deal with frictional constraints, thus avoiding the linearization of the friction cone [31]. The FC test in this paper uses the following lemma [13].

Lemma 1: Consider a grasp with associated sets $G$ and $W$, and let $\mathcal{I}$ be the set of strictly interior points of $\mathrm{CH}(W)$ and $H_{l}$ be a supporting hyperplane of $\mathrm{CH}(W)$, i.e., a hyperplane containing the facet $l$ of $\mathrm{CH}(W)$. The origin $O$ of the wrench space satisfies $O \in \mathcal{I}$ if and only if $\forall l O$ and any point $P \in \mathcal{I}$ lie in the same half-space defined by $H_{l}$.

In this paper, the condition of Lemma 1 is checked by choosing the centroid of the primitive contact wrenches in $W$, which is always an interior point of $\mathrm{CH}(W)$, as point $P$. Fig. 1 illustrates the concept with an FC grasp and a non-FC grasp for the 3-D wrench space of a 2-D object (note that the test is general, and therefore, also valid for the 6-D wrench space associated with a 3-D object). Here, $\mathrm{CH}(W)$ is represented by simplicial facets, i.e., each facet has the minimum possible number of vertices $(d$ for a $d$-dimensional space) [32].

To quantify the goodness of a grasp, the considered grasp quality measure, which is one of the most common, is the largest perturbation wrench that the grasp can resist independently of the perturbation direction [15]. Assuming without loss of generality that the maximum allowed grasping force is a unitary 


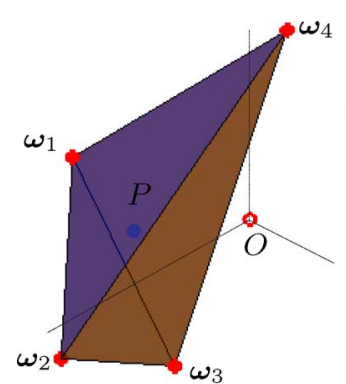

(a) (b)

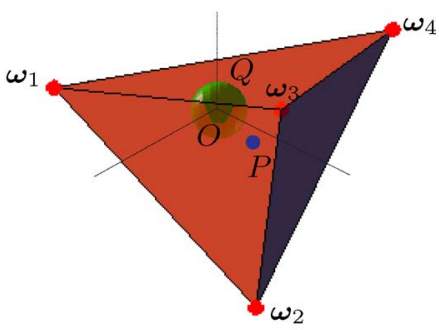

Fig. 1. FC test. (a) Non-FC grasp. Hyperplane formed by $\left\{\boldsymbol{\omega}_{2}, \boldsymbol{\omega}_{3}, \boldsymbol{\omega}_{4}\right\}$ leaves $P$ and $O$ in different half-spaces. (b) FC grasp. All the supporting hyperplanes of $\mathrm{CH}(W)$ leave $P$ and $O$ in the same half-space. The radius $Q$ of the largest inscribed sphere indicates the grasp quality.

force along $\hat{\boldsymbol{n}}_{i j}$, this grasp quality is equivalent to the radius of the largest hypersphere centered on $O$ and fully contained in $\mathrm{CH}(W)$, i.e., it is the distance from $O$ to the closest facet of $\mathrm{CH}(W)$ [see Fig. 1(b)].

\section{COMputation of a SuItable Starting FC GrasP}

The synthesis of a suitable starting FC grasp for the search of the ICRs is performed in two phases: The first generates an initial grasp with uncontrolled quality, and the second uses it to generate a grasp with locally optimal quality. The approach is based on that in [13], but more efficient procedures are given here. The following sections describe the two phases and provide a comparison with previous works.

\section{A. Search for an Initial FC Grasp}

Basically, the procedure to search for an initial FC grasp with $n$ points randomly chooses a set $G_{\text {aux }}$ of $n-1$ points from $\Omega$, and then determines the region of the wrench space where the $n$th point must be located in order to generate an FC grasp (the necessary and sufficient condition provided in [9] is used for this purpose). If there is at least one point of $\Omega$ in this region, an FC grasp is directly obtained; otherwise, one of the points in $G_{\text {aux }}$ is iteratively replaced following certain rules until a solution is found. The detailed algorithm is as follows.

Algorithm 1: Search for an initial FC grasp

1) Generate a random set $G_{\text {aux }}=\left\{\boldsymbol{\omega}_{1}, \ldots, \boldsymbol{\omega}_{n-1}\right\}$

2) Build $W_{\text {aux }}=\left\{\boldsymbol{\omega}_{11}, \ldots, \boldsymbol{\omega}_{1 m}, \ldots, \boldsymbol{\omega}_{n-1}, \ldots, \boldsymbol{\omega}_{n-1 m}\right\}$ $\cup\{O\}$

3) Compute $\mathrm{CH}\left(W_{\text {aux }}\right)$

4) Determine the $L$ supporting hyperplanes $H_{l}$ of $\mathrm{CH}\left(W_{\text {aux }}\right)$ that contain $O, l=1 \ldots L$. Let $H_{l}^{+}$and $H_{l}^{-}$be the two half-spaces defined by $H_{l}$, with $\mathrm{CH}\left(W_{\text {aux }}\right) \subset H_{l}^{-}$

5) If $L=0$, then (i.e., $G_{\text {aux }}$ is already an FC grasp)

a) Randomly choose $\boldsymbol{\omega}_{c} \notin G_{\text {aux }}$

b) Go to Step 9

6) Find the sets of wrenches

$$
\begin{aligned}
& \mathcal{C}_{1}=\left\{\boldsymbol{\omega}_{i} \mid \boldsymbol{\omega}_{i 1} \vee \ldots \vee \boldsymbol{\omega}_{i m} \in \bigcap_{l} H_{l}^{+}\right\} \text {and } \\
& \mathcal{C}_{2}=\left\{\boldsymbol{\omega}_{j} \mid \boldsymbol{\omega}_{j 1} \wedge \ldots \wedge \boldsymbol{\omega}_{j m} \in \bigcap_{l} H_{l}^{-}\right\}
\end{aligned}
$$

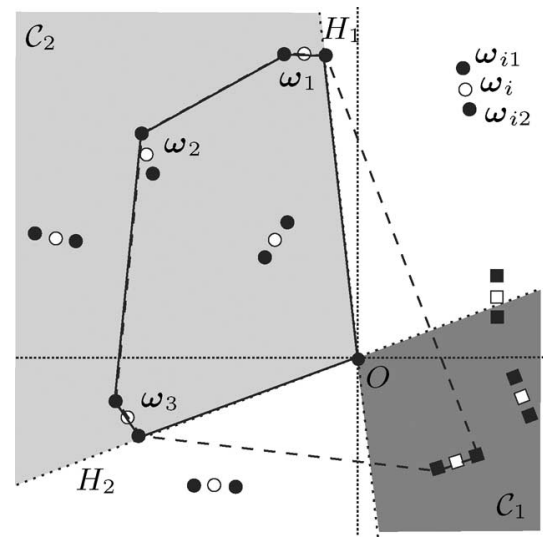

Fig. 2. Synthesis of an FC grasp. The convex hull for the grasp set $W_{\text {aux }}=$ $\left\{\boldsymbol{\omega}_{1}, \boldsymbol{\omega}_{2}, \boldsymbol{\omega}_{3}\right\} \cup\{\boldsymbol{0}\}$ (in continuous lines) defines the supporting hyperplanes $H_{1}$ and $H_{2}$ containing the origin. The convex region defining the set $\mathcal{C}_{1}$ (in dark gray) contains primitive wrenches corresponding to three points (depicted as squares, while the other wrenches are represented by circles). Thus, the algorithm provides three FC grasps; the convex hull corresponding to one of them is illustrated in discontinuous lines. The convex region defining the set $\mathcal{C}_{2}$ is shown in light gray.

7) If $\mathcal{C}_{1}=\emptyset$, then

a) Randomly pick $\boldsymbol{\omega}_{l}$ such that $\boldsymbol{\omega}_{l} \notin \mathcal{C}_{2}$

b) Find $\boldsymbol{\omega}_{i} \in G_{\text {aux }}$ closest to $\boldsymbol{\omega}_{l}$

c) Update $G_{\text {aux }}$ by replacing $\boldsymbol{\omega}_{i}$ with $\boldsymbol{\omega}_{l}$

d) Go to Step 2

8) Randomly choose $\boldsymbol{\omega}_{c} \in \mathcal{C}_{1}$

9) Form $G=G_{\text {aux }} \cup\left\{\boldsymbol{\omega}_{c}\right\}=\left\{\boldsymbol{\omega}_{1}, \ldots, \boldsymbol{\omega}_{n-1}, \boldsymbol{\omega}_{c}\right\}$

10) Return $C=\left\{\boldsymbol{p}_{1}, \ldots, \boldsymbol{p}_{i}, \ldots, \boldsymbol{p}_{n}\right\}$ such that $\boldsymbol{p}_{i}$ is a contact point associated with $\boldsymbol{\omega}_{i} \in G$

Fig. 2 illustrates the synthesis of an FC grasp for a hypothetical 2-D wrench space. The algorithm ends when one FC grasp is obtained and returns it, but it may provide as many FC grasps as points belong to $\mathcal{C}_{1}$ in the last iteration. When $\mathcal{C}_{1}$ is empty, the $n-1$ points associated with the wrenches in $G_{\text {aux }}$ will never yield an FC grasp. Thus, when this happens, the combination of wrenches in the current $G_{\text {aux }}$ is left out for subsequent searches (in Step 7c) to progressively explore the search space and ensure the completeness of the algorithm (this was not included in the aforementioned algorithm to simplify its structure).

To illustrate the procedure with a real example, the search for a four-finger FC grasp of a discretized ellipse using frictionless contacts is shown in Fig. 3 (for 2-D objects the wrench space is 3-D, allowing a graphical representation). Fig. 3(a) shows the discretized ellipse with the neighboring points connected by a segment and three fingers randomly placed on the ellipse boundary. Fig. 3(b) shows the wrench space and $\mathrm{CH}\left(W_{\text {aux }}\right)$ (the convex hull of the corresponding wrenches plus the origin $O$ ), the supporting hyperplanes $H_{1}, H_{2}$, and $H_{3}$ containing the origin $O$, the regions defining the sets $\mathcal{C}_{1}$ and $\mathcal{C}_{2}$, and the 14 wrenches contained in $\mathcal{C}_{1}$. The corresponding 14 points on the ellipse boundary, together with an FC grasp obtained by randomly choosing one of these 14 points for the fourth contact, are illustrated in Fig. 3(c). Fig. 3(d) shows the convex hull for the selected grasp and the largest inscribed sphere centered on $O$, whose radius represents the grasp quality $Q$. 


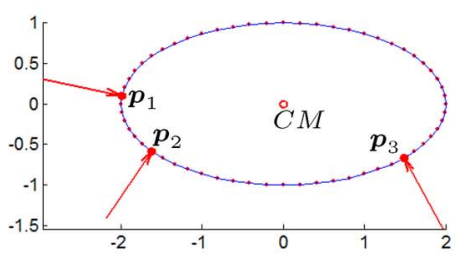

(a)

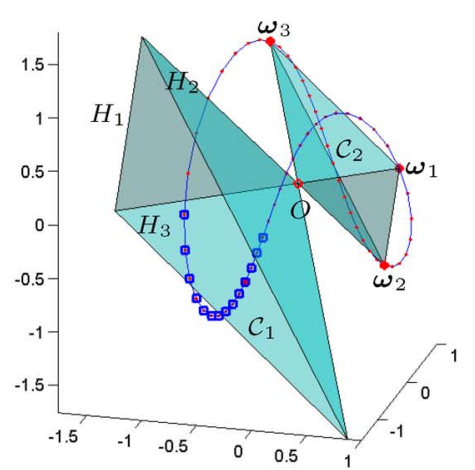

(b)

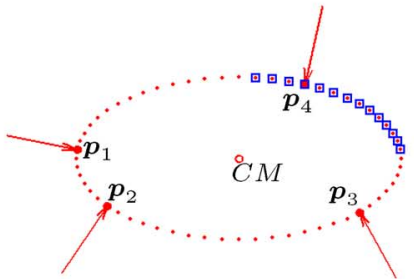

(c)

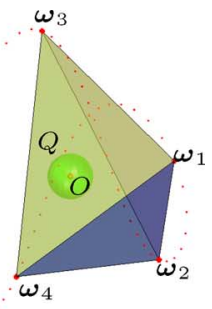

(d)

Fig. 3. Search for an initial FC grasp with four frictionless contacts on a discretized ellipse. (a) Ellipse with three contacts randomly selected. (b) Convex hull of $W_{\text {aux }}=\left\{\boldsymbol{\omega}_{1}, \boldsymbol{\omega}_{2}, \boldsymbol{\omega}_{3}\right\} \cup\{O\}$ with the supporting hyperplanes $H_{1}, H_{2}$, and $H_{3}$ containing the origin $O$ and defining the region $\mathcal{C}_{1}$, and the 14 points $\boldsymbol{\omega}_{i}$ lying in the region $\mathcal{C}_{1}$. (c) Corresponding 14 points $\boldsymbol{p}_{i}$ on the ellipse and an FC grasp obtained by randomly selecting one of them as $\boldsymbol{p}_{4}$. (d) Convex hull of the obtained FC grasp $G=\left\{\boldsymbol{\omega}_{1}, \boldsymbol{\omega}_{2}, \boldsymbol{\omega}_{3}, \boldsymbol{\omega}_{4}\right\}$; the largest inscribed sphere indicates the grasp quality $Q=0.197$.

Algorithm 1 has a heuristic nature; nevertheless, some remarks about its computational cost can be made. Step 3 requires the computation of a 6-D convex hull. This paper uses the qhull implementation, which has a complexity $\mathcal{O}\left(N v^{2} / 6\right)$, with $v$ the number of vertices in the convex hull [32]. Step 6 requires the classification of the $N-n+1$ points in $\Omega$ with respect to the $L$ supporting hyperplanes $H_{l}$, which are described by linear equations. The total number of iterations required to find an FC grasp (or to decide that there is no solution at all) depends on the random choices of $G_{\text {aux }}$ in Step 1 and $\boldsymbol{\omega}_{l}$ in Step 7a.

\section{B. Search for a Locally Optimal FC Grasp}

The procedure to search for a locally optimal grasp uses Algorithm 1 to generate an initial FC grasp. Then, the facet of the corresponding convex hull that limits the grasp quality is identified, and one of its vertices (primitive wrenches associated with a contact point) is iteratively replaced to look for a better grasp. This is done by computing the region of the wrench space where the new wrench must be located in order to increase the grasp quality and then by looking for a primitive wrench in this region. If one is found, the associated contact point replaces the existing one. If no replacement is found for any of the vertices of the facet, the current grasp is returned as a locally optimal one. The considered grasp quality is equivalent to the largest perturbation wrench that the grasp resists, independently of its direction (see Section II-C). The detailed algorithm is as follows.

Algorithm 2: Search for a locally optimal grasp

1) Use Algorithm 1 to find an initial FC grasp and build the wrench sets $G=\left\{\boldsymbol{\omega}_{1}, \ldots, \boldsymbol{\omega}_{n}\right\}, W=$ $\left\{\boldsymbol{\omega}_{11}, \ldots, \boldsymbol{\omega}_{1 m}, \ldots, \boldsymbol{\omega}_{n 1}, \ldots, \boldsymbol{\omega}_{n m}\right\}$

2) Compute $\mathrm{CH}(W)$ and the corresponding grasp quality $Q$. Let $F_{Q}$ be the facet of $\mathrm{CH}(W)$ determining $Q$

3) Find the set $G_{\text {aux }} \subset G$ including the wrenches $\boldsymbol{\omega}_{i}$ with at least one primitive wrench $\boldsymbol{\omega}_{i j}$ in $F_{Q}$, i.e., $G_{\text {aux }}=$ $\left\{\boldsymbol{\omega}_{i} \mid \boldsymbol{\omega}_{i} \in G \wedge \exists \boldsymbol{\omega}_{i j} \in F_{Q}\right\}$. Let $J \leq n$ be the number of elements in $G_{\text {aux }}$

4) For $i=1$ to $J$ (i.e., for each $\boldsymbol{\omega}_{i} \in G_{\text {aux }}$ ), do

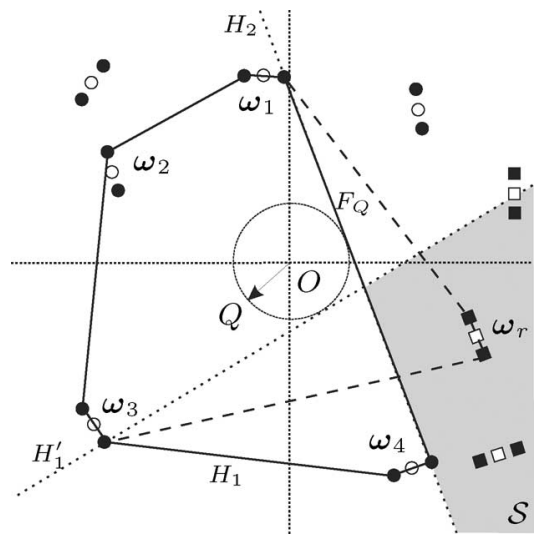

Fig. 4. Optimization of an initial FC grasp with $G=\left\{\boldsymbol{\omega}_{1}, \boldsymbol{\omega}_{2}, \boldsymbol{\omega}_{3}, \boldsymbol{\omega}_{4}\right\} . F_{Q}$ is determined by $\boldsymbol{\omega}_{1 j}$ and $\boldsymbol{\omega}_{4 k}$, and then, $G_{\mathrm{aux}}=\left\{\boldsymbol{\omega}_{1}, \boldsymbol{\omega}_{4}\right\}$. To replace $\boldsymbol{\omega}_{4}$, hyperplanes $H_{1}$ and $H_{2}$ give rise to hyperplanes $H_{1}^{\prime}$ and $H_{2}^{\prime}=H_{2}\left(F_{Q} \subset\right.$ $\mathrm{H}_{2}$ ) defining the region $\mathcal{S}$ (gray area) containing the set $\mathcal{C}$ of replacement candidates (depicted as squares). $\boldsymbol{\omega}_{r}$ is chosen to replace $\boldsymbol{\omega}_{4}$ and generate the new grasp with improved quality, and $G=\left\{\boldsymbol{\omega}_{1}, \boldsymbol{\omega}_{2}, \boldsymbol{\omega}_{3}, \boldsymbol{\omega}_{r}\right\}$ (the modified sides of $\mathrm{CH}(W)$ are shown in dashed lines).

a) For each supporting hyperplane $H_{l}$ of $\mathrm{CH}(W)$ containing at least one primitive wrench of $\boldsymbol{\omega}_{i}$ (i.e., $\exists j \mid$ $\boldsymbol{\omega}_{i j} \in H_{l} \wedge \boldsymbol{\omega}_{i j} \in F_{Q}$ ) build the hyperplane $H_{l}^{\prime}$ at a distance $Q$ from the origin $O$ passing through the primitive wrenches of $H_{l}$ that do not belong to $\boldsymbol{\omega}_{i}$ (i.e., passing through $\boldsymbol{\omega}_{h j} \mid \boldsymbol{\omega}_{h j} \in H_{l}$ and $h \neq i$ ), and leaving $O$ and $\boldsymbol{\omega}_{i}$ in different half-spaces

b) Consider the region $\mathcal{S}=\bigcap H_{l}^{\prime+}$, with $H_{l}^{\prime+}$ the halfspace defined by $H_{l}^{\prime}$ such that $O \notin H_{l}^{\prime+}$. Find the set $\mathcal{C}$ of wrenches with at least one primitive wrench in $\mathcal{S}$ (i.e., $\mathcal{C}=\left\{\boldsymbol{\omega}_{i} \mid \boldsymbol{\omega}_{i 1} \vee \ldots \vee \boldsymbol{\omega}_{i m} \in \mathcal{S}\right\}$ )

c) If $\mathcal{C} \neq \emptyset$, then

i) Update $G$ by replacing $\boldsymbol{\omega}_{i}$ with a random $\boldsymbol{\omega}_{r} \in \mathcal{C}$

ii) Update $W$ according to $G$

iii) Go to Step 2

5) Return $C=\left\{\boldsymbol{p}_{1}, \ldots, \boldsymbol{p}_{i}, \ldots, \boldsymbol{p}_{n}\right\}$ such that $\boldsymbol{p}_{i}$ is a contact point associated with $\boldsymbol{\omega}_{i} \in G$ 


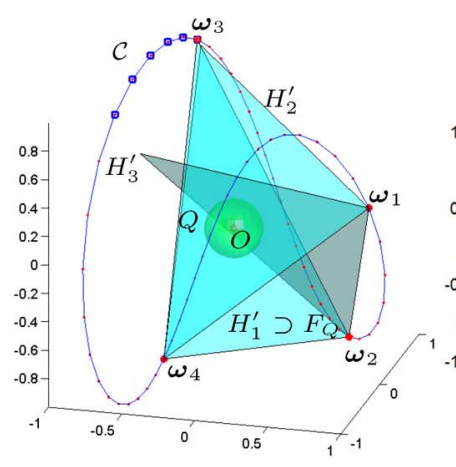

(a)

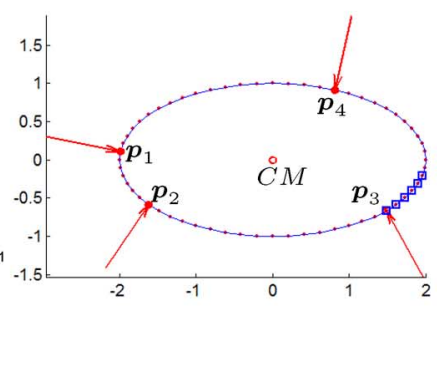

(b)

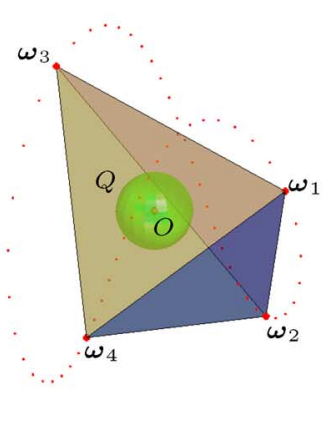

(c)

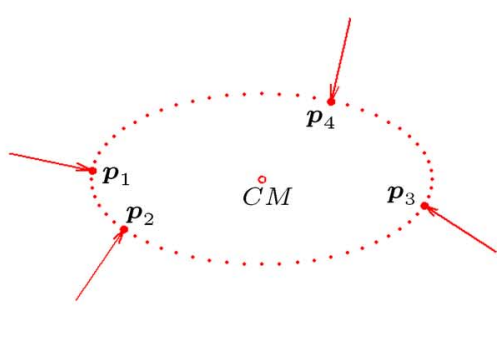

(d)

Fig. 5. Search for the locally optimal grasp. Replacement of $\boldsymbol{p}_{3}$ in the initial FC grasp in Fig. 3(d). (a) Hyperplanes $H_{l}^{\prime}$ tangent to the sphere of radius $Q=0.197$ defining the set $\mathcal{C}$ of replacement candidates (depicted as dark squares) that improve the actual grasp quality. (b) Corresponding contact points on the ellipse. (c) $\mathrm{CH}(W)$ for the new grasp obtained after the replacement of $\boldsymbol{\omega}_{3}$, with grasp quality $Q=0.266$. (d) New grasp on the ellipse.

Fig. 4 illustrates the procedure for a hypothetical 2-D wrench space. Note that Algorithm 2 does not involve an explicit FC test, which reduces the computational complexity when compared to previous works [13], [33]. Besides, only one computation of $\mathrm{CH}(W)$ is required in each iteration of the algorithm. The total number of iterations required to reach the local maximum depends on the initial FC grasp and on the number of local maxima in the wrench space.

To illustrate the procedure with a real example, Fig. 5 represents the first iteration of the locally optimal grasp search for the ellipse and the starting FC grasp in Fig. 3. The starting grasp quality $Q$ is determined by primitive wrenches of the contacts at $\boldsymbol{p}_{2}, \boldsymbol{p}_{3}$, and $\boldsymbol{p}_{4}$, which gives $G_{\text {aux }}=\left\{\boldsymbol{\omega}_{2}, \boldsymbol{\omega}_{3}, \boldsymbol{\omega}_{4}\right\}$. To replace $\boldsymbol{\omega}_{3}$, the hyperplanes $H_{1}^{\prime}, H_{2}^{\prime}$, and $H_{3}^{\prime}$ are built at a distance $Q$ from the origin (i.e., they are tangent to the sphere of radius $Q$ centered on $O$ ), as shown in Fig. 5(a). The set $\mathcal{C}$ contains five wrenches that improve the actual grasp quality, highlighted with squares in the wrench space in Fig. 5(a), while their corresponding five contact points on the ellipse boundary are highlighted in Fig. 5(b). A random replacement for $\omega_{3}$ is selected from $\mathcal{C}$, and Fig. 5(c) shows the resulting convex hull for the new grasp with the inscribed sphere indicating the new grasp quality. Fig. 5(d) shows the new grasping points on the ellipse.

\section{Comparison With Previous Works}

Previous work has already addressed the problem of FC grasp synthesis on discretized 3-D objects. One approach generates a number of concurrent FC grasps with four frictional fingers but without any optimality criterion, leaving to the user the choice of the most suitable grasp for a particular task [34]. The algorithm is based on the location of regions in the 3-D physical space where the axes of the friction cones seem to intersect. For each region, random subsets of four grasping points are tested for the FC condition by choosing an arbitrary point in the region and testing whether it is included in the four friction cones. The algorithm is capable of computing hundreds of FC grasps; the reported running times required to obtain one FC grasp in objects described with 2000 surface points are below $2 \mathrm{~s}$ on a 2.4-GHz PC.
Another approach that is able to deal with frictional and frictionless contacts starts with the random selection of $n$ contact points and then iteratively moves the points to reduce the distance between the convex hull of the applied wrenches and the origin of the wrench space [12]. When the procedure is trapped in a local minimum, the set of points is divided into subsets by using a separating hyperplane in the wrench space, and the FC search is decomposed into simpler subproblems. The algorithm uses an FC test in each iteration that implies the solution of a linear programming problem based on the ray-shooting technique [29]. The algorithm is complete in the sense that it finds an FC grasp if it exists in the discrete sampling of the surface, but it does not ensure any optimality.

Algorithm 1 also looks for the FC grasp in the wrench space, it is applicable to any number of frictional or frictionless fingers, and progressively covers the search space until an FC grasp is found or until the nonexistence of an FC grasp is proved, thus being complete. This algorithm is based on a random selection of points, while the approach in [12] performs a search checking all the combinations of neighboring points to decrease an objective function (the distance between the convex hull and the origin of the wrench space). Hence, no formal comparison in terms of computational complexity is evident. Nevertheless, the following remarks may help in a qualitative comparison of both approaches. Algorithm 1 does not include an explicit FC test in each iteration since the FC condition is embedded in the search process itself. Besides, the algorithm may return more than one FC grasp (depending on the number of wrenches lying in the subset $\mathcal{C}_{1}$ ). If necessary, a first selection according to any quality measure could therefore be done at this point. However, Algorithm 1 requires an explicit convex hull computation in each iteration, which may be fast for frictionless contacts but is more time-consuming for frictional contacts. The performance of Algorithm 1 may be further improved if a good heuristic to guide the random selection of points is found. On the other hand, in both approaches, the efficiency depends on the initial contact points, which are randomly chosen. Therefore, some criterion to initially choose points spread on the object surface could improve the search time (ideas with related potential applications are given in [35]). 
If a grasp of higher quality is desired (as in the case of ICR computation), a locally optimal grasp can be obtained from an initial one with Algorithm 2. Some works have already tackled the synthesis of optimal grasps for 3-D objects with surfaces described by appropriate parameters [10], [36], [37], but these works cannot be applied to discrete objects because they solve optimization problems using continuous variables. For discrete objects, an algorithm for fixture synthesis was proposed based on the minimization of the workpiece positioning error due to uncertainties in the locators position and in the workpiece geometry [38]. This algorithm finds a locally optimal fixture, but the approach is not applicable to frictional contacts. A previous work of Roa and Suárez [13] laid the basis for the algorithms presented here. Algorithm 1 was improved using the necessary and sufficient FC condition in [9], which speeds up the time required to obtain the first FC grasp. In Algorithm 2, points are selected by choosing only candidates that improve the actual grasp quality, thus eliminating the need for an explicit FC test for each candidate and improving the time required to obtain the locally optimal grasp.

\section{COMPUTATION OF ICRS}

\section{A. ICRs From a Given FC Grasp}

This section presents the procedure to compute ICRs such that if each finger is located at any point inside its corresponding ICR, an FC grasp with a desired minimum grasp quality is always obtained. ICRs can be computed starting from any given FC grasp. However, since the quality of this starting grasp will bound the reachable maximum minimum quality, if a high minimum quality is desired, then the use of a locally optimal grasp obtained with Algorithm 2 in Section III-B is recommended. A high starting grasp quality will also result in larger ICRs.

Basically, the procedure works as follows. Given a starting FC grasp with quality $Q_{s}$, the desired minimum grasp quality $Q_{r}=\alpha Q_{s}$ (with $0<\alpha \leq 1$ ) for any FC grasp within the ICRs is selected; when $\alpha \rightarrow 0$, the ICRs allow FC grasps with no lower limit on the grasp quality (note that $Q_{r}=0$ is actually a forbidden value as it does not ensure the FC condition, namely that any possible $\mathrm{CH}(W)$ will strictly contain the origin $O$ ). The larger the $Q_{r}$, the smaller the ICRs. Therefore, $Q_{r}$ must be selected as a tradeoff between the desired robustness of potential grasps to external perturbations and the flexibility or error margin in finger positioning on the object surface. Once $Q_{r}$ is fixed, a set of hyperplanes in the wrench space parallel to the facets of the $\mathrm{CH}(W)$ of the starting grasp and tangent to a hypersphere of radius $Q_{r}$ is used to determine regions of the wrench space where new wrenches (associated with new contacts) will generate FC grasps with quality $Q \geq Q_{r}$. Finally, depending on whether each ICR is constrained to be a continuous region or not, a neighboring condition of the physical points associated with the new valid wrenches can be imposed. The detailed algorithm follows.

Algorithm 3: Determination of the ICRs

1) Find a starting FC grasp $C=\left\{\boldsymbol{p}_{1}, \ldots, \boldsymbol{p}_{n}\right\}$ using Algorithm 2, and obtain the corresponding set $W=$ $\left\{\boldsymbol{\omega}_{11}, \ldots, \boldsymbol{\omega}_{1 m}, \ldots, \boldsymbol{\omega}_{n 1}, \ldots, \boldsymbol{\omega}_{n m}\right\}$ and its quality $Q_{s}$

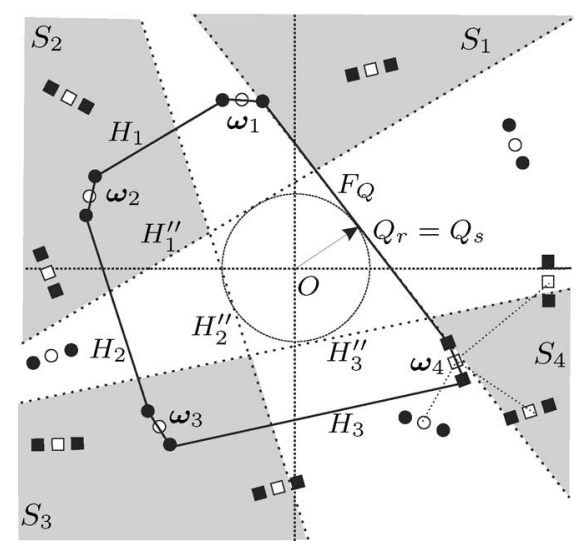

Fig. 6. Search for ICRs ensuring a minimum grasp quality. Search zones $S_{i}$ for each grasping point are depicted in gray, and the wrenches associated with neighboring points within each ICR are depicted with squares.

2) Select the minimum acceptable quality $Q_{r}=\alpha Q_{s}$, with $0<\alpha \leq 1$

3) Compute $\mathrm{CH}(W)$

4) For $i=1$ to $n$ (i.e., for each contact point $\boldsymbol{p}_{i} \in C$ ), do

a) For each facet $F_{k}$ of $\mathrm{CH}(W)$ having at least one vertex $\boldsymbol{\omega}_{i j}$, build the hyperplane $H_{k}^{\prime \prime}$ parallel to $F_{k}$ and at a distance $Q_{r}$ from the origin $O$, leaving $O$ and $F_{k}$ in different half-spaces. Let $H_{k}^{\prime \prime+}$ be the open half-space such that $\boldsymbol{\omega}_{i j} \in H_{k}^{\prime \prime+}$

b) Initialize $\mathrm{ICR}_{i}=\left\{\boldsymbol{p}_{i}\right\}$

c) Label $\boldsymbol{p}_{i}$ as open

d) While there are open points $\boldsymbol{p}_{h} \in \mathrm{ICR}_{i}$, do

i) For all the neighboring points $\boldsymbol{p}_{s}$ of $\boldsymbol{p}_{h}$, do If $\exists j$ such that $\forall k \boldsymbol{\omega}_{s j} \in H_{k}^{\prime \prime+}$, then

$\mathrm{ICR}_{i}=\mathrm{ICR}_{i} \cup\left\{\boldsymbol{p}_{s}\right\}$

Label $\boldsymbol{p}_{s}$ as open

ii) Label $\boldsymbol{p}_{h}$ as closed

5) Return the ICRs

Fig. 6 illustrates the procedure for a hypothetical 2-D wrench space; note that due to the geometrical construction, any physical point $\boldsymbol{p}_{h}$ with a primitive wrench $\boldsymbol{\omega}_{h j}$ in the region $S_{i}=\bigcap_{k} H_{k}^{\prime \prime+}$ can replace the point $\boldsymbol{p}_{i}$ of the given initial FC grasp without losing the FC property and providing a quality $Q \geq Q_{r}$. Algorithm 3 is computationally very simple, the core being Step 4 . The determination of the hyperplanes $H_{k}^{\prime \prime}$ parallel to $F_{k}$ and at a distance $Q_{r}$ from $O$ in Step 4 a is quite simple and straightforward, as is the determination of $H_{k}^{\prime \prime+}$. Step $4 \mathrm{~d}$ is the most costly step because it is necessary to check whether an unknown number of primitive wrenches $\boldsymbol{\omega}_{h j}$ of an unknown number of points $\boldsymbol{p}_{h}$ belongs to each half-space $H_{k}^{\prime \prime+}$. Nevertheless, each of these tests is just an evaluation of the sign of a linear equation in the 6-D wrench space.

The procedure is illustrated with a real example in Fig. 7 using the four-finger grasp with frictionless contacts of the discrete ellipse. The starting FC grasp is shown on the ellipse (physical space) in Fig. 7(a) and in the wrench space in Fig. 7(b); it is the locally optimal grasp obtained with Algorithm 2 for the example in Fig. 5. Fig. 7(c) shows the hyperplanes $H_{k}^{\prime \prime}, k=1, \ldots, 4$ tangent to the sphere of radius $Q_{r}=0.5 Q_{s}$, the search zones 


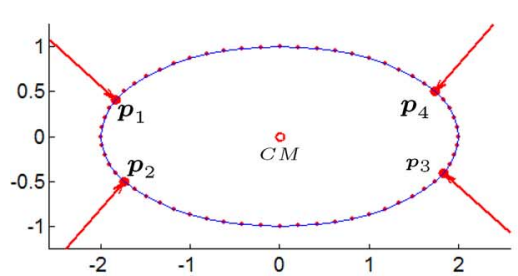

(a)

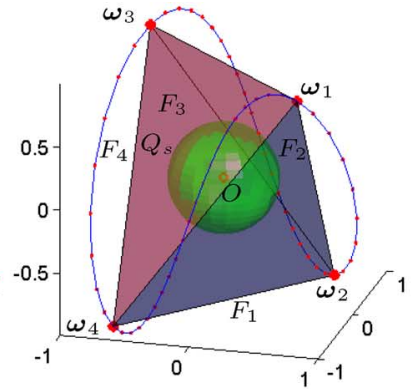

(b)

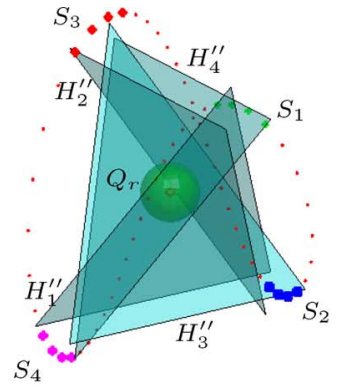

(c)

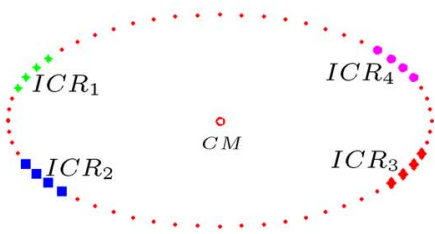

(d)

Fig. 7. Search for the ICRs for a discretized ellipse. (a) Starting locally optimal FC grasp on the ellipse. (b) Starting FC grasp in the wrench space, with grasp quality $Q_{s}=0.426$. (c) Search zones $S_{i}$ defined by the hyperplanes $H_{k}^{\prime \prime}$ and wrenches within each $S_{i}$ for $Q_{r}=0.213(\alpha=0.5)$. (d) ICRs on the ellipse.

$S_{i}, i=1, \ldots, 4$, and the wrenches associated with the contact points in the ICRs. The obtained ICRs can be seen on the ellipse in Fig. 7(d).

The number of points in each ICR varies depending on the smoothness of the surface around the contact points of the initial FC grasp (i.e., the rate of change of the surface normal direction around the contact points) and, of course, on the detail in the representation of the object surface. Note that the ICRs depend on the starting grasp; the search for the largest ICRs (or optimal in any sense) is not addressed in this paper, but it is an interesting issue to explore as future work.

A variation of Algorithm 3 can be obtained by removing the neighborhood condition in Step 4(d)i, thus considering all the points $\boldsymbol{p}_{h}$ with at least one primitive wrench $\boldsymbol{\omega}_{h j}$ inside the search zone $S_{i}$ as part of $\mathrm{ICR}_{i}$. This would yield ICRs that may be composed of discontinuous regions on the object boundary, since the wrenches $\boldsymbol{\omega}_{h j}$ might be close in the wrench space, but the points $\boldsymbol{p}_{h}$ might not be neighbors at all on the object boundary.

\section{B. ICRs for Grasps With Some Contacts Fixed Beforehand}

A particular extension of the proposed approach deals with the search of ICRs for contact points that fully constrain the object when a given number of contacts is fixed beforehand, for instance, when there exist several fixed locator pins to hold a workpiece or when an object lies on a surface (which predefines some contacts on the object). If $r$ contact points are provided, the approach can be used to determine the missing $n-r$ contacts and ICRs to guarantee the FC condition. Algorithms 1 and 2 remain essentially unchanged when applied to this particular case; they are only adjusted to prevent the points fixed beforehand from changing in any iteration.

The ICRs for the fixed contact points are not required (it is assumed that their locations are precisely determined), and therefore, they do not need to be computed; this is reflected in some changes in Step 4a of Algorithm 3. In the original algorithm, Step 4a builds several hyperplanes $H_{k}^{\prime \prime}$ tangent to the hypersphere of radius $Q_{r}$ and parallel to each facet $F_{k}$ of $\mathrm{CH}(W)$ having at least one vertex $\boldsymbol{\omega}_{i j}$. In order to search the ICRs for one missing contact point $\boldsymbol{p}_{i}$, this step builds several hyperplanes tangent to the hypersphere of radius $Q_{r}$ and con-

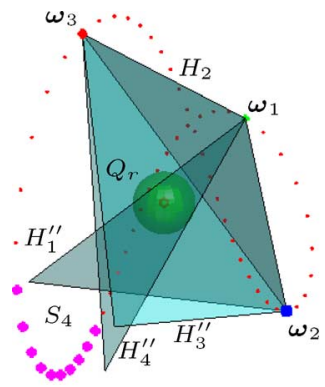

(a)

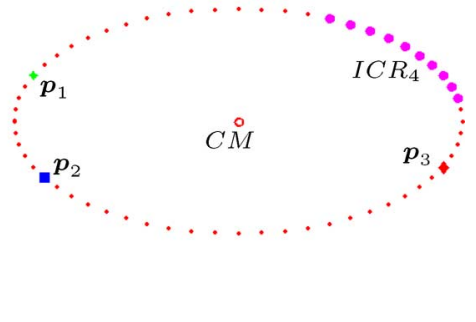

(b)
Fig. 8. Search of the ICRs for a discretized ellipse with three contacts fixed beforehand. (a) Hyperplanes $H_{1}^{\prime \prime}, H_{3}^{\prime \prime}$, and $H_{4}^{\prime \prime}$ are tangent to the sphere of radius $Q_{r}=0.5 Q_{s}=0.213$ and define the search zone $S_{4}$. (b) ICR for the point $\boldsymbol{p}_{4}$ on the ellipse.

taining vertices $\boldsymbol{\omega}_{i j}$ belonging to $\boldsymbol{p}_{i}$. This modified procedure is illustrated in Fig. 8 using the starting grasp shown in Fig. 7(a) and (b). Assuming the points $\boldsymbol{p}_{1}, \boldsymbol{p}_{2}$, and $\boldsymbol{p}_{3}$ to be fixed beforehand, the ICR for the point $\boldsymbol{p}_{4}$ was computed. Fig. 8(a) shows the hyperplanes $H_{1}^{\prime \prime}, H_{3}^{\prime \prime}$, and $H_{4}^{\prime \prime}$ that define the search zone $S_{4}$. The ICR for the point $\boldsymbol{p}_{4}$ is illustrated in Fig. 8(b). Note that when there exist points fixed beforehand, larger ICRs are obtained for the rest of the contacts.

\section{Comparison With Previous Works}

ICR computation for general 3-D objects based on initial examples was originally proposed to compute a number of new grasps that preserve some properties of a single starting example of a successful grasp [23]. The paper considers as grasp quality the inverse of the sum of the normal components of the forces required to balance the worst-case external expected wrench in a given task [39]. In the approach proposed here, the grasp quality measure used is the largest perturbation wrench that the grasp can resist, irrespective of the perturbation direction [15], which is a task-independent measure. On the other hand, both approaches are applicable to any number of fingers, and the results depend on the choice of the starting example, which is provided here with a locally optimal grasp.

Regarding ICR computation when $r$ contact points are fixed beforehand, a procedure to compute an optimal location for the $n-r$ missing points formulates the problem as a set of 


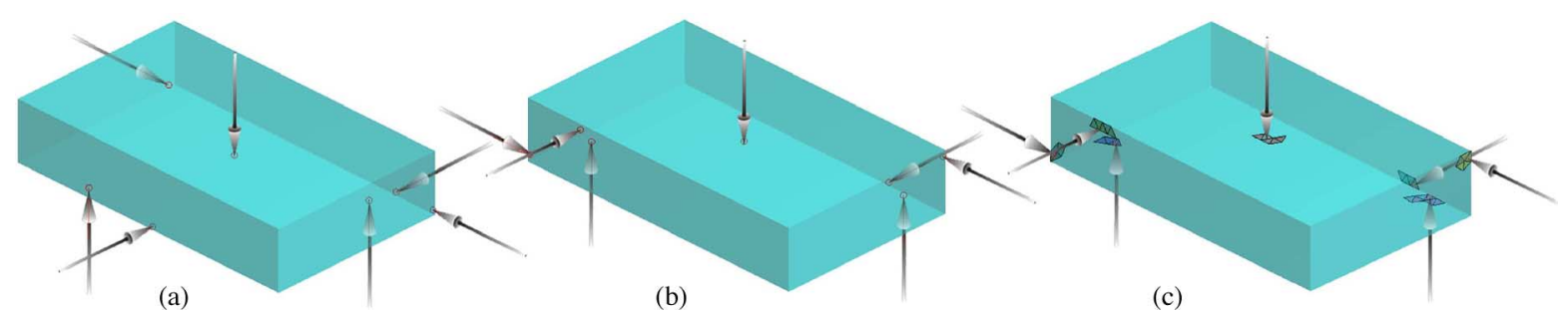

Fig. 9. Example 1. Seven-finger frictionless grasp of a parallelepiped. (a) Initial FC grasp, $Q=0.0098$ (Algorithm 1). (b) Locally optimal FC grasp, $Q=0.292$ (Algorithm 2). (c) ICRs for each finger, $Q_{r}=0.219$ (Algorithm 3 with $\alpha=0.75$ ).

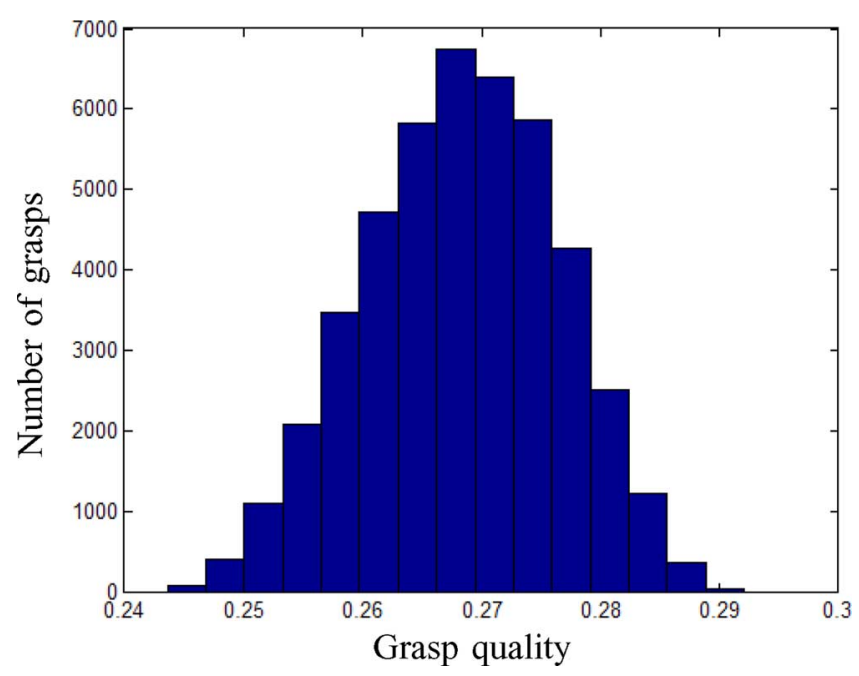

Fig. 10. Histogram for Example 1 with the quality distribution for all possible grasps within the ICRs for $Q_{r}=0.219(\alpha=0.75)$.
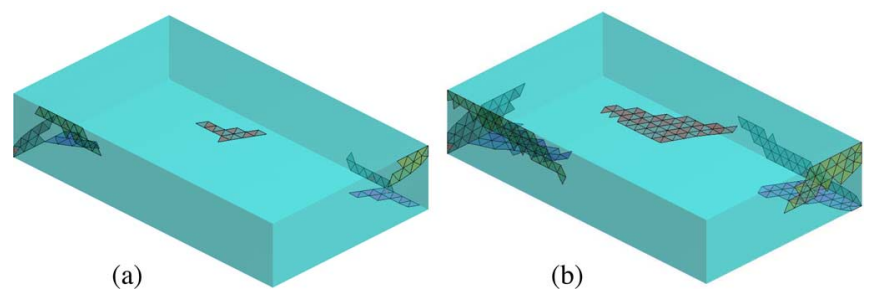

Fig. 11. ICRs for Example 1 with a minimum quality of (a) $Q_{r}=0.146(\alpha=$ $0.5)$. (b) $Q_{r} \approx 0\left(\alpha=10^{-5}\right)$.

inequalities solved with nonlinear programming techniques [9]. The quality measure used is the $L_{2}$ distance between the CM of the object and the centroid of the contact points [8]. The procedure can also be used to compute the ICR for one grasping point, considering that the other $n-1$ points are fixed. However, the procedure can only be applied when the 3-D object is described by a set of parameters (e.g., a polyhedron or an object with parameterized curved surfaces) and requires some points to be necessarily fixed beforehand, i.e., it is not applicable in a general case where all $n$ fingers must be located on the object. In contrast, the use of parameterized surfaces allows the computation of continuous ICRs. The approach proposed here (using Algorithms 1, 2, and 3) solves the general problem of locating the $r$ missing points and computing their corresponding ICRs for any discrete object.
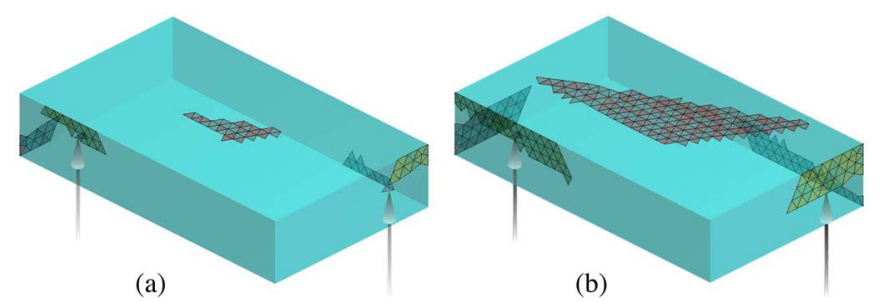

Fig. 12. ICRs for Example 1 with 2 contacts fixed beforehand (shown as arrows). (a) $Q_{r}=0.146(\alpha=0.5)$. (b) $Q_{r} \approx 0\left(\alpha=10^{-5}\right)$.

TABLE I

RESUlts FOR A DifFERENT NuMBER OF POINTS ON THE OBJECT BOUNDARY

\begin{tabular}{cccrcrr}
$N$ & $t_{Q i}[s]$ & $Q_{i}$ & $t_{Q f}[s]$ & $Q_{f}$ & $t_{I C R}[s]$ & grasps \\
\hline 1628 & 0.54 & 0.030 & 6.73 & 0.20 & 0.089 & 493 \\
3422 & 0.36 & 0.031 & 13.23 & 0.21 & 0.179 & 47432 \\
\hline
\end{tabular}

\section{APPLICATION EXAMPLES}

The proposed approach has been implemented using MAT$\mathrm{LAB}$ on a Pentium IV 3.2-GHz PC. The performance of the algorithms is illustrated with different 3-D objects whose boundaries are described by triangular meshes. The potential contact points $\boldsymbol{p}_{i}$ are the centroids of the triangles in the mesh, and the corresponding surface normal directions are the directions normal to the triangles. Two points are considered as neighbors if their corresponding triangles share an edge.

\section{A. Example 1: Seven Frictionless Contacts on a Polyhedron}

The first example uses a parallelepiped described with a mesh of 3422 triangles. This simple object was chosen to allow an easy intuitive interpretation of the results (more complex objects are presented in subsequent examples). The example presents the computation of ICRs for a grasp (or fixture) with seven frictionless contacts.

The first FC grasp obtained with Algorithm 1 is shown in Fig. 9(a). The time required to obtain it was $0.49 \mathrm{~s}$ in five iterations (in the fifth iteration, Algorithm 1 provides another 18 possible FC grasps), and the grasp quality is 0.0098 . The locally optimal FC grasp [see Fig. 9(b)] was obtained with Algorithm 2 in $24.3 \mathrm{~s}$ and 48 iterations, and the locally optimal grasp quality is 0.2921 , which means a quality improvement factor of 29.8 (i.e., the ratio between the quality of the optimized and the initial FC grasp). Note that each iteration of Algorithms 1 and 2 involves the computation of one convex hull. Fig. 9(c) illustrates the corresponding ICRs obtained with Algorithm 3 in $0.17 \mathrm{~s}$ using 


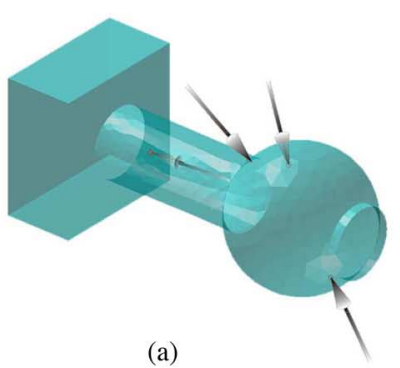

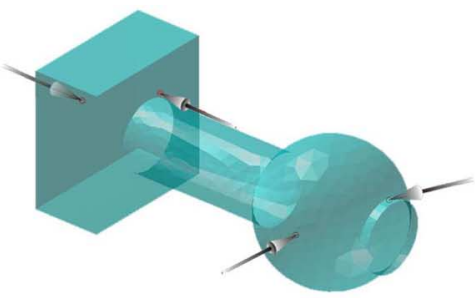

(b)

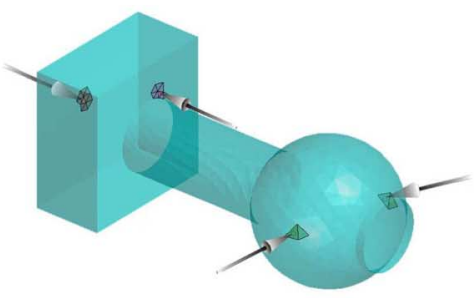

(c)

Fig. 13. Example 2. Frictional grasp of a workpiece. (a) Initial FC grasp, $Q=0.035$ (Algorithm 1). (b) Locally optimal FC grasp, $Q=0.174$ (Algorithm 2). (c) ICRs for each finger, $Q_{r}=0.131$ (Algorithm 3 with $\alpha=0.75$ ).

a minimum acceptable quality of $Q_{r}=0.219(\alpha=0.75)$. The points within the ICRs can be combined to generate 45000 different grasps whose quality distribution is shown in Fig. 10. For smaller $Q_{r}$ (i.e., smaller $\alpha$ ), the ICRs become larger, as illustrated in Fig. 11 for the same starting grasp but assuming $Q_{r}=0.146(\alpha=0.5)$ and $Q_{r} \approx 0\left(\alpha=10^{-5} \approx 0\right)$.

Consider now that in the optimal grasp in Fig. 9(b), the two contacts on the bottom of the parallelepiped have been precisely fixed beforehand (it may seem to be an artificial assumption, but it is used here to allow direct comparison of the results). Using Algorithm 3 with the modifications described in Section IV-B gives the ICRs in Fig. 12. Note that for the same minimum quality $Q_{r}$, the ICRs for the other five contacts are larger than the corresponding ones in Fig. 11.

In order to illustrate the performance of the approach for a different number of points $N$ in the object model, for this example, the algorithms were run using a set $\Omega$ with $N=1628$ and $N=3422$ points. The averaged results for 50 trials for each case are given in Table I, which includes the times required to obtain the first FC grasp $\left(t_{Q i}\right)$, the locally optimal grasp $\left(t_{Q f}\right)$, and the ICRs $\left(t_{\mathrm{ICR}}\right)$, as well as the corresponding qualities for the initial $\left(Q_{i}\right)$ and optimal $\left(Q_{f}\right)$ grasps and the number of possible grasps allowed by the ICRs (grasps).

\section{B. Example 2: Four Frictional Contacts on a Nonpolyhedral Object}

In this example, a workpiece presented in [10] and discretized with 3946 triangles is to be grasped using four frictional contacts and a friction coefficient $\mu=0.2$ (see Fig. 13). The friction cone has been linearized with an eight-side polyhedral convex cone. Algorithm 1 provides the first FC grasp [see Fig. 13(a)] in $2.3 \mathrm{~s}$ in the first iteration with grasp quality 0.035 . Algorithm 2 provides the locally optimal grasp [see Fig. 13(b)] in $114 \mathrm{~s}$ after 29 iterations, with a locally optimal grasp quality of 0.174 , which means a quality improvement factor of 5 . Algorithm 3 computes the ICRs for $Q_{r}=0.131(\alpha=0.75)$ in $66 \mathrm{~s}$. The points within the ICRs allow 320 different grasps, whose quality distribution can be seen in Fig. 14.

The relevance of the goodness of the starting grasp is illustrated in Fig. 15, which shows the ICRs computed using Algorithm 3 starting with the initial and the locally optimal FC grasp in Fig. 13(a) and (b), respectively, and $Q_{r}=0.01$ (note that $Q_{r}$ must be lower than the quality of the initial grasp). The ICRs allow 14196 and 509490 different FC grasps, respectively.

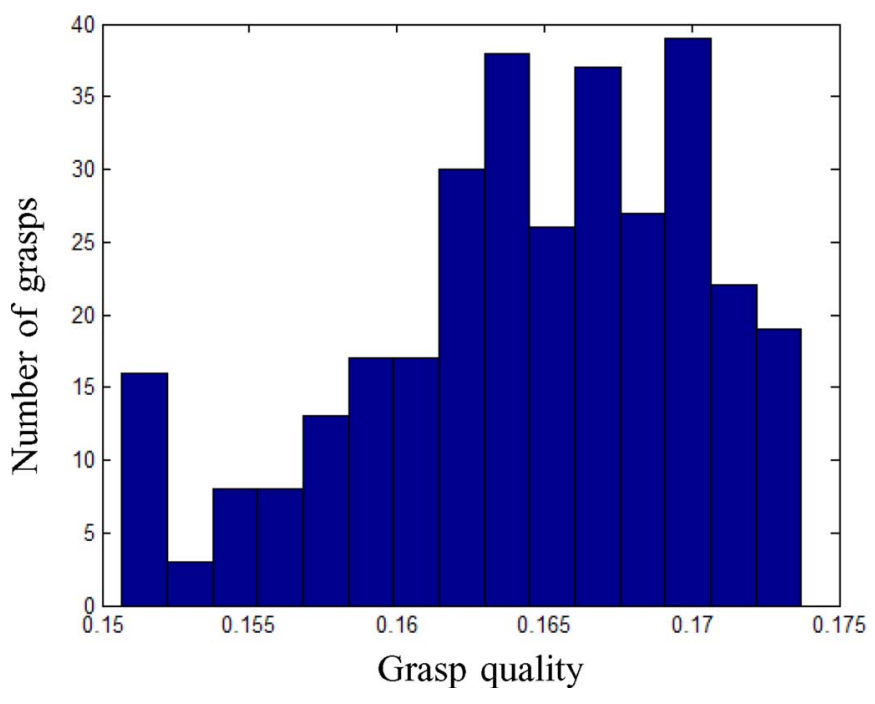

Fig. 14. Grasp quality distribution for all possible grasps within the ICRs for Example 2 with $Q_{r}=0.131(\alpha=0.75)$.
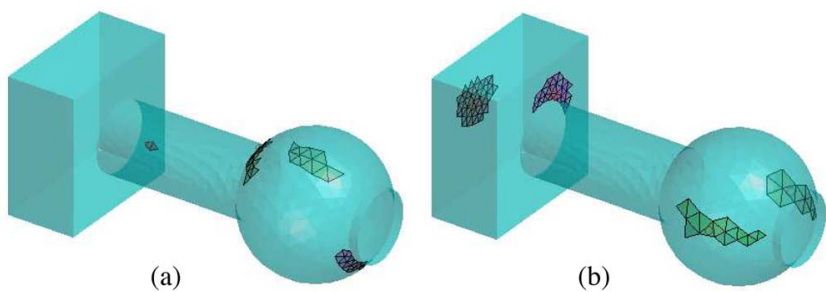

Fig. 15. ICRs for Example 2 with the same minimum quality $Q_{r}=0.01$ but computed from (a) the initial grasp and (b) the locally optimal grasp.

Even when the ICRs ensure the same minimum quality in both cases, in the second one, the size of the ICRs allows a larger number of possible FC grasps, thus justifying the search for the ICRs based on a starting grasp of higher quality.

For this example, the algorithms were run using a different number of faces $m$ in the discretized approximation of the friction cone. The averaged results for 50 trials for 4,8 , and 12 friction cone faces are summarized in Table II.

In order to show the effect of the neighboring condition on the ICRs computation, Fig. 16(a) shows the ICRs for $Q_{r} \approx 0(\alpha=$ $10^{-5}$ ) considering neighboring points, and, Fig. 16(b) shows the ICRs obtained for the same $Q_{r}$ but using the variation of Algorithm 3 described at the end of Section IV-A that neglects 

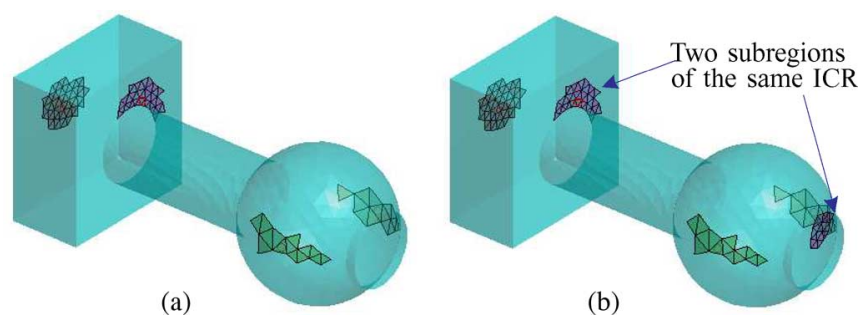

Fig. 16. ICRs for Example 2 with $Q_{r} \approx 0\left(\alpha=10^{-5}\right)$ using (a) Algorithm 3 and (b) Algorithm 3, but dropping the condition of neighboring points.

TABLE II

RESUltS FOR A DifFERENT NUMBER OF FACES IN THE DisCRETIZED FRICTION CONE

\begin{tabular}{cccrcrr}
$m$ & $t_{Q i}[s]$ & $Q_{i}$ & $t_{Q f}[s]$ & $Q_{f}$ & $t_{I C R}[s]$ & grasps \\
\hline 4 & 0.57 & 0.071 & 29.73 & 0.128 & 8.82 & 4424 \\
8 & 2.38 & 0.081 & 92.50 & 0.149 & 69.07 & 6034 \\
12 & 6.88 & 0.080 & 196.08 & 0.145 & 316.91 & 9077 \\
\hline
\end{tabular}

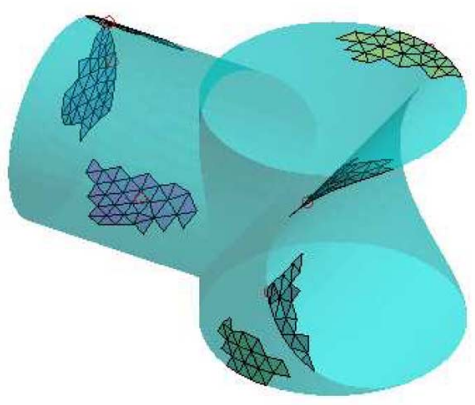

(a)

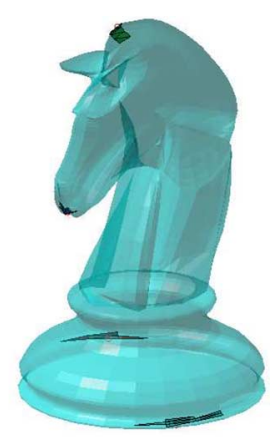

(b)
Fig. 17. ICRs for other figures, with $Q_{r} \approx 0$ using (a) 7-finger frictionless contacts and (b) 4-finger frictional contacts.

the neighboring condition and, therefore, causes some ICRs to split into disjoint zones.

\section{Example 3: Seven Frictionless Contacts on a Nonpolyhedral Object}

Fig. 17(a) shows the ICRs obtained for another nonpolyhedral workpiece discretized with 3222 triangles considering $Q_{r} \approx$ $0\left(\alpha=10^{-5}\right)$ and a grasp with seven frictionless contacts.

\section{Example 4: Four Frictional Contacts on an Irregular Object}

Fig. 17(b) shows the ICRs obtained for a highly irregular object, a chess knight, discretized with 4750 triangles considering $Q_{r} \approx 0\left(\alpha=10^{-5}\right)$ and a grasp with four frictional contacts.

\section{E. Different Number of Fingers on a Nonpolyhedral Object}

In order to illustrate the performance of the approach for a different number of fingers $n$ on the same object, the algorithms were run for the nonpolyhedral object of Example 2 using four, five, and six fingers. The averaged results for 30 trials for each case are summarized in Table III.
TABLE III

RESULTS FOR A DIFFERENT NUMBER OF FINGERS

\begin{tabular}{rccrcrr}
$n$ & $t_{Q i}[s]$ & $Q_{i}$ & $t_{Q f}[s]$ & $Q_{f}$ & $t_{I C R}[s]$ & grasps \\
\hline 4 & 2.38 & 0.081 & 92.50 & 0.149 & 69.07 & 6034 \\
5 & 2.90 & 0.118 & 193.83 & 0.180 & 218.28 & 71708 \\
6 & 3.66 & 0.174 & 307.35 & 0.240 & 602.93 & 1007154 \\
\hline
\end{tabular}

\section{CONCLUSIONS AND FUTURE WORK}

This paper presents an approach for the computation of ICRs to perform grasps using frictional or frictionless contacts and ensuring a minimum grasp quality for any number $n$ of fingers, provided that $n \geq 3$ for frictional contacts and $n \geq 7$ for frictionless contacts. Procedures to compute an initial FC grasp and to look for a locally optimal one are also provided. The algorithms are implemented, and the execution results, as well as the examples included in the paper, illustrate the relevance and efficiency of the approach, which uses geometric reasonings based on the information and structure of the wrench space and can be applied to search ICRs, starting from any provided FC grasp. In addition, the approach is extended to compute the ICRs when some contact locations are fixed beforehand.

There are several directions for future research based on the approach proposed in the paper. The first is related to the computation or validation of the set of points $\Omega$ describing the object boundary, such that the FC property can be formally assured between the neighboring points in the ICRs. In this paper, it is assumed that the set $\Omega$ is well-conditioned and dense enough to be useful, which seems to be valid for practical applications. If the object is described by a triangular mesh, instead of using only the centroids of the triangles, a potential solution is the evaluation of the force at each vertex of a triangle to ensure the FC at any point in the triangle. A related topic is the development of an algorithm to properly sample the object surface for grasp planning applications. Another future research line focuses on improving the computational time of the approach. In this sense, the development of heuristics or criteria to guide the random selections of points in Steps 1 and 8 in Algorithm 1 and Step 4(c)i in Algorithm 2 are potential sources of improvement (the selection of grasping points properly spread on the object surface may help in this sense). Finally, a relevant future work from the application point of view is the use of ICRs to plan regrasping tasks for manipulation purposes.

\section{APPENDIX}

\section{ON THE Metric of THE WrenCh SpaCe AND ITS INFLUENCE ON THE GRASP QUALITY MEASURE}

Planning in robotics usually involves the use of the configuration space, which collects position and orientation information, and the wrench space, which collects force and torque information. If the process involves the use of Euclidean distances in these spaces, their metrics must be properly adjusted to avoid unit inconsistency in the distance computation. The grasp quality used in this paper [15] is a Euclidean distance in the wrench space, and therefore, the metric of the wrench space is adjusted using a parameter $\rho$ with units of length to define the wrenches 
as $\boldsymbol{\omega}=(\boldsymbol{f} \boldsymbol{\tau} / \rho)^{T}$. In this way, all the components of $\boldsymbol{\omega}$ have units of force, and the grasp quality can be computed using the metric $L_{2}$. Nevertheless, if $\rho$ is constant and the modules of the finger forces $\boldsymbol{f}_{i} i=1, \ldots, n$ are kept constant, when the object is scaled, the grasp quality will change accordingly (from 0 up to a limit imposed by the convex hull of the grasp forces $\boldsymbol{f}_{i}$, $i=1, \ldots, n)$. In order to avoid this effect, $\rho$ is defined as a function of the object size, and two approaches are presented in this line. One approach considers $\rho$ as the largest distance from the object's CM to any point of the object. Thus, the maximum potential torque is equal to the maximum applied force (typically unitary), which creates a nice scaled wrench space but does not have a real physical meaning. The other approach considers $\rho$ as the radius of gyration of the object, which has a physical meaning in terms of energy, as follows. Let $\boldsymbol{p}$ be the object position, $\phi$ the object orientation, $m$ the object mass, and $I$ the object inertia around a (given) rotation axis (note that $I=\rho^{2} m$ ). If the metric of the configuration space is properly adjusted defining a configuration as $\boldsymbol{c}=(\boldsymbol{p} \rho \boldsymbol{\phi})^{T}$, then the kinetic energy of the object can be computed as

$$
\begin{aligned}
E_{K} & =\frac{1}{2} m\|\dot{\boldsymbol{p}}\|^{2}+\frac{1}{2} I\|\dot{\boldsymbol{\phi}}\|^{2} \\
& =\frac{1}{2} m\left(\|\dot{\boldsymbol{p}}\|^{2}+\|\rho \dot{\boldsymbol{\phi}}\|^{2}\right)=\frac{1}{2} m\|\dot{\boldsymbol{c}}\|^{2}
\end{aligned}
$$

i.e., $\rho \phi$ is equivalent to a translational dimension. If the wrench is adjusted as $\boldsymbol{\omega}=(\boldsymbol{f} \boldsymbol{\tau} / \rho)^{T}$, the work $W$ needed to move the object an amount $\Delta c$ under a wrench $\omega$ is

$$
W=\boldsymbol{w}^{T} \Delta \boldsymbol{c}=\boldsymbol{f}^{T} \Delta \boldsymbol{p}+\left(\frac{\boldsymbol{\tau}}{\rho}\right)^{T} \rho \Delta \boldsymbol{\phi}=\boldsymbol{f}^{T} \Delta \boldsymbol{p}+\boldsymbol{\tau}^{T} \Delta \boldsymbol{\phi}
$$

which, as can be seen, is equivalent to the real work. In these equations, we assume the knowledge of the rotation axis to illustrate the concepts in a simple way, but for 3-D objects, the equations can be formulated considering rotations around the reference axes and the proper metric adjustments for each one.

We prefer this last metric adjustment for the wrench space, but even when the value of the grasp quality measure depends on the metric, the algorithms presented in the paper are still valid.

\section{REFERENCES}

[1] A. Bicchi, "On the closure properties of robotic grasping," Int. J. Robot. Res., vol. 14, no. 4, pp. 319-344, 1995.

[2] A. Miller, S. Knoop, H. Christensen, and P. Allen, "Automatic grasp planning using shape primitives," in Proc. IEEE ICRA, 2003, pp. 18241829.

[3] C. Goldfeder, P. Allen, C. Lackner, and R. Pelossof, "Grasp planning via decomposition trees," in Proc. IEEE ICRA, 2007, pp. 4679-4684.

[4] Y. Liu, "Computing n-finger form-closure grasps on polygonal objects," Int. J. Robot. Res., vol. 19, no. 2, pp. 149-158, 2000.

[5] J. Cornellà and R. Suárez, "Efficient determination of four-point formclosure optimal constraints of polygonal objects," IEEE Trans. Autom. Sci. Eng., vol. 6, no. 1, pp. 121-130, Jan. 2009.

[6] I. Chen and J. Burdick, "Finding antipodal point grasps on irregularly shaped objects," IEEE Trans. Robot. Autom., vol. 9, no. 4, pp. 507-512, Aug. 1993.

[7] N. Niparnan and A. Sudsang, "Computing all force-closure grasps of 2D objects from contact point set," in Proc. IEEE/RSJ IROS, 2006, pp. 15991604.
[8] J. Ponce, S. Sullivan, A. Sudsang, J. Boissonat, and J. Merlet, "On computing four-finger equilibrium and force-closure grasps of polyhedral objects," Int. J. Robot. Res., vol. 16, no. 1, pp. 11-35, 1997.

[9] D. Ding, Y. Liu, and S. Wang, "Computation of 3-D form-closure grasps," IEEE Trans. Robot. Autom., vol. 17, no. 4, pp. 515-522, Aug. 2001.

[10] X. Zhu and J. Wang, "Synthesis of force-closure grasps on 3-D objects based on the Q distance," IEEE Trans. Robot. Autom., vol. 19, no. 4 , pp. 669-679, Aug. 2003.

[11] X. Zhu and H. Ding, "Planning force-closure grasps on 3-D objects," in Proc. IEEE ICRA, 2004, pp. 1258-1263.

[12] Y. Liu, M. Lam, and D. Ding, "A complete and efficient algorithm for searching 3-D form closure grasps in the discrete domain," IEEE Trans. Robot., vol. 20, no. 5, pp. 805-816, Oct. 2004.

[13] M. Roa and R. Suarez, "Finding locally optimum force-closure grasps," J. Robot. Comput. Integr. Manuf., vol. 25, no. 3, pp. 536-544, 2009.

[14] J. Ponce and B. Faverjon, "On computing three-finger force-closure grasps of polygonal objects," IEEE Trans. Robot. Autom., vol. 11, no. 6, pp. 868 881, Dec. 1995.

[15] C. Ferrari and J. Canny, "Planning optimal grasps," in Proc. IEEE ICRA 1992, pp. 2290-2295.

[16] Z. Li and S. Sastry, "Task-oriented optimal grasping by multifingered robotic hands," IEEE J. Robot. Autom., vol. 4, no. 1, pp. 32-44, Feb. 1988.

[17] C. Borst, M. Fischer, and G. Hirzinger, "Grasp planning: How to choose a suitable task wrench space," in Proc. IEEE ICRA, 2004, pp. 319325 .

[18] K. Shimoga, "Robot grasp synthesis algorithms: A survey," Int. J. Robot. Res., vol. 15, no. 3, pp. 230-266, 1996.

[19] M. Roa, R. Suarez, and J. Cornella, "Medidas de calidad para la prensión de objetos," Rev. Iberoamericana de Autom. e Inf. Ind., vol. 5, no. 1, pp. 66-82, 2008 .

[20] A. Bicchi and V. Kumar, "Robotic grasping and contact: A review," in Proc. IEEE ICRA, 2000, pp. 348-352.

[21] A. Okamura, N. Smaby, and M. Cutkosky, "An overview of dexterous manipulation," in Proc. IEEE ICRA, 2000, pp. 255-262.

[22] V. Nguyen, "Constructing force-closure grasps," Int. J. Robot. Res., vol. 7, no. 3, pp. 3-16, 1988.

[23] N. Pollard, "Closure and quality equivalence for efficient synthesis of grasps from examples," Int. J. Robot. Res., vol. 23, no. 6, pp. 595-614, 2004.

[24] J. Cornellà and R. Suárez, "Determining independent grasp regions on 2D discrete objects," in Proc. IEEE/RSJ IROS, 2005, pp. 2936-2941.

[25] M. Roa and R. Suarez, "Independent contact regions for frictional grasps on 3D objects," in Proc. IEEE ICRA, 2008, pp. 1622-1627.

[26] X. Markenscoff, L. Ni, and C. Papadimitriou, "The geometry of grasping," Int. J. Robot. Res., vol. 9, no. 1, pp. 61-74, 1990.

[27] B. Mishra, J. Schwartz, and M. Sharir, "On the existence and synthesis of multifinger positive grips," Algorithmica, vol. 2, no. 4, pp. 541-558, 1987.

[28] R. Murray, Z. Li, and S. Sastry, A Mathematical Introduction to Robotic Manipulation. Boca Raton, FL: CRC, 1994.

[29] Y. Liu, "Qualitative test and force optimization of 3-D frictional formclosure grasps using linear programming," IEEE Trans. Robot. Autom., vol. 15 , no. 1, pp. 163-173, Feb. 1999.

[30] X. Zhu, H. Ding, and S. Tso, "A pseudodistance function and its applications," IEEE Trans. Robot. Autom., vol. 20, no. 2, pp. 344-352, Apr. 2004.

[31] L. Han, J. Trinkle, and Z. Li, "Grasp analysis as linear matrix inequality problems," IEEE Trans. Robot. Autom., vol. 16, no. 6, pp. 663-674, Dec. 2000.

[32] C. Barber, D. Dobkin, and H. Huhdanpaa, "The quickhull algorithm for convex hulls," ACM Trans. Math. Softw., vol. 22, no. 4, pp. 469-483, 1996.

[33] M. Roa and R. Suarez, "Geometrical approach for grasp synthesis on discretized 3D objects," in Proc. IEEE/RSJ IROS, 2007, pp. 32833288.

[34] N. Niparnan and A. Sudsang, "Fast computation of 4-fingered forceclosure grasps from surface points," in Proc. IEEE/RSJ IROS, 2004, pp. 3692-3697.

[35] R. Suarez and J. Rosell, "Searching form-closure fixturing points on objects described by triangular meshes," in Proc. IEEE Int. Symp. Assem. Manuf. (ISAM), 2007, pp. 7-12.

[36] Y. Zheng and W. Qian, "An enhanced ray-shooting approach to forceclosure problems," ASME J. Manuf. Sci. Eng., vol. 128, no. 4, pp. 960968, 2006. 
[37] Y. Zheng and W. Qian, "On some weakness existing in optimal grasp planning," Mech. Mach. Theory, vol. 43, no. 1, pp. 576-590, 2008.

[38] M. Wang and D. Pelinescu, "Optimizing fixture layout in a point-set domain," IEEE Trans. Robot. Autom., vol. 17, no. 3, pp. 312-323, Jun. 2001.

[39] X. Zhu, H. Ding, and H. Li, "A quantitative measure for multifingered grasps," in Proc. IEEE/ASME Int. Conf. Adv. Intell. Mechatron., 2001, pp. 213-219.

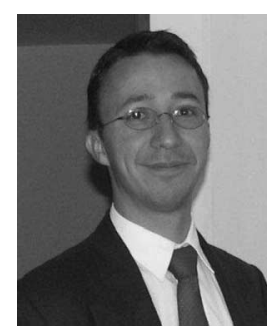

Máximo A. Roa (S'03) received the B.S. degree in mechanical engineering and the M.S. degree in industrial automation (cum laude) from the National University of Colombia, Bogota, Colombia, in 1998 and 2004 , respectively. He is currently working toward the Ph.D. degree in robotics and advanced automation with the Technical University of Catalonia, Barcelona, Spain.

From 2000 to 2008, he was a Researcher and an Assistant Professor with the Department of Mechanical and Mechatronic Engineering, National University of Colombia. He is currently a Research and Development Engineer with the Large Format Printing Division, Hewlett Packard. His current research interests include grasping and manipulation, mechanical hands, telemanipulation, and walking robots.

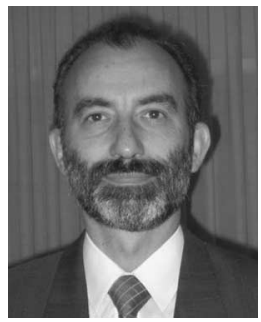

Raúl Suárez (M'96) received the Electronic Engineer degree (with honors) from the National University of San Juan, San Juan, Argentina, in 1984 and the Ph.D. degree (cum laude) from the Technical University of Catalonia (UPC), Barcelona, Spain, in 1993.

$\mathrm{He}$ is currently a Researcher with the Institute of Industrial and Control Engineering, UPC, where he has been responsible for the research line "Process Control" (1998-2003) and, at present, he is the Deputy Director (since 2003) and the Coordinator of the Doctoral Programs "Advanced Automation and Robotics" (since 1995) and "Automatic Control, Robotics, and Computer Vision" (since 2006). His current research areas include grasping and manipulation, mechanical hands, fixturing, assembly, task planning, telemanipulation, and manufacturing automation. 